\title{
The Modified Glasgow Prognostic Score Is an Independent Prognostic Factor in Patients with Inoperable Thoracic Esophageal Squamous Cell Carcinoma Undergoing Chemoradiotherapy
}

\author{
Peng Zhang ${ }^{*}$, Mian $\mathrm{Xi}^{{ }^{*}}$, Qiao-Qiao Li1 ${ }^{1}$ Li-Ru He${ }^{1}$, Shi-Liang Liu ${ }^{1}$, Lei Zhao ${ }^{1}$, Jing-Xian Shen², \\ Meng-Zhong Liu ${ }^{\circledR}$ \\ 1. Sun Yat-sen University Cancer Center; State Key Laboratory of Oncology in South China, Collaborative Innovation Center for Cancer Medicine; \\ Department of Radiation Oncology, Cancer Center, Sun Yat-sen University, Guangzhou 510060, People's Republic of China \\ 2. Sun Yat-sen University Cancer Center; State Key Laboratory of Oncology in South China, Collaborative Innovation Center for Cancer Medicine; \\ Imaging Diagnosis and Interventional Center, Cancer Center, Sun Yat-sen University, Guangzhou 510060, People's Republic of China \\ *These authors contributed equally to this work.
}

$\triangle$ Corresponding author: Meng-Zhong Liu, MD, Department of Radiation, Sun Yat-sen University Cancer Center, 651 Dongfeng Road East, Guangzhou, Guangdong 510060, P. R. China. Tel.: +86-20-87343649 Fax: +86-20-87343649 Email: liumengzhong@126.com.

() Ivyspring International Publisher. This is an open-access article distributed under the terms of the Creative Commons License (http://creativecommons.org/ licenses/by-nc-nd/3.0/). Reproduction is permitted for personal, noncommercial use, provided that the article is in whole, unmodified, and properly cited.

Received: 2014.05.04; Accepted: 2014.07.04; Published: 2014.09.06

\begin{abstract}
Background and purpose: There is increasing evidence that the presence of an inflammation-based prognostic score (modified Glasgow prognostic score, mGPS) is associated with survival in patients with advanced cancer. This study aimed to assess whether the mGPS has prognostic value in patients with thoracic esophageal squamous cell carcinoma undergoing chemoradiotherapy.

Materials and methods: A total of 212 patients undergoing chemoradiotherapy for newly-diagnosed esophageal squamous cell carcinoma between October, 2006 and December, 20II were retrospectively analyzed. Serum C-reactive protein (CRP) and albumin were measured before initiation of treatment. The relationships between the $\mathrm{mGPS}$ and other relevant variables including white blood cell count, neutrophilic granulocyte count, platelet count, hemoglobin, bilirubin, aspartate aminotransferase (AST), alanine aminotransferase (ALT) and lactate dehydrogenase (LDH) were analyzed. Overall survival (OS) and progression-free survival (PFS) were calculated. Significant prognostic factors were identified using univariate and multivariate analyses.

Results: Three-year OS for all patients was $24.6 \%$; 3 -year PFS was $21.3 \%$. Patients with a mGPS of 0 , I and 2 were 90, 78, 44, respectively. Higher mGPS was related to higher white blood cell, neutrophilic granulocyte and platelet counts, and lower total bilirubin. T stage, $M$ stage and mGPS were independent prognostic indicators for OS; T stage, $M$ stage, mGPS and platelet count were independent prognostic indicators for PFS.

Conclusions: Pretreatment mGPS is an easily measurable significant prognostic factor and can be used in combination with conventional TNM staging to predict survival in patients with squamous cell carcinoma undergoing chemoradiotherapy.
\end{abstract}

Key words: esophageal cancer, chemoradiotherapy, mGPS, prognostic factor, survival.

\section{Introduction}

Esophageal cancer is one of the most lethal tumor types, with a 5 -year survival rate of only $17 \% .^{1}$ Esophageal cancer is usually diagnosed at an ad- vanced stage and only $30-40 \%$ of cases are suitable for curative surgical resection. ${ }^{2}$ Chemoradiotherapy is the mainstay treatment in patients for whom surgery is 
not an option. There is increasing evidence that the presence of a systemic inflammatory response is a prognostic indicator in patients with esophageal cancer. ${ }^{3-5}$ The acute phase proteins albumin and C-reactive protein (CRP) are indicators of a systemic inflammatory response. ${ }^{6-7}$ The modified Glasgow prognostic score (mGPS) combines albumin and CRP into a risk stratification score for predicting clinical outcome in patients with cancer. Previous reports have shown that the mGPS is an indicator of the prognosis of patients with cancer of the digestive tract, including colorectal cancer, ${ }^{8}$ gastric cancer ${ }^{9}$ and pancreatic cancer ${ }^{10}$. However, few studies have reported the prognostic value of the mGPS in patients with esophageal squamous cell carcinoma (SCC), especially patients undergoing chemoradiotherapy.

The purpose of this study was to evaluate the potential prognostic value of the mGPS in terms of long-term outcome in patients with inoperable thoracic esophageal SCC receiving chemoradiotherapy. The relationship between the mGPS and other relevant variables such as white blood cell count, neutrophilic granulocyte count, platelet count, hemoglobin, aspartate aminotransferase (AST), alanine aminotransferase (ALT), bilirubin and lactate dehydrogenase (LDH) was assessed. The value of these factors for predicting the survival of patients with inoperable thoracic esophageal SCC undergoing chemoradiotherapy was analyzed.

\section{Materials and Methods}

\section{Clinical data and patient groups}

Between October, 2006 and December, 2011, the medical records of 212 patients who were diagnosed with pathologically-proven esophageal SCC at Sun Yat-sen University Cancer Center in China were reviewed retrospectively. All patients had either refused surgery or were unable to undergo surgery. The pretreatment work-up included a complete medical history, physical examination, computed tomography (CT) scans of the chest and abdomen, barium esophagography and endoscopic ultrasonography. The patients were staged using the $6^{\text {th }}$ edition of the AJCC staging system. The diagnosis was pathologically confirmed as SCC in all cases. All patients underwent regular follow-up at the same hospital. The date of last follow-up was April, 2013.

To evaluate the mGPS, blood test results from the day before treatment (or no more than 1 week before treatment) were used. The mGPS is based on both CRP and albumin. Patients who had both elevated CRP (> $10 \mathrm{mg} / \mathrm{L})$ and hypoalbuminurea $(<35$ $\mathrm{g} / \mathrm{L})$ were assigned a score of 2 . Patients with only elevated CRP (> $10 \mathrm{mg} / \mathrm{L}$ ) were assigned a score of 1 .
Patients with neither of these abnormalities were assigned a score of $0 .{ }^{11}$

\section{Radiotherapy and chemotherapy}

External beam radiotherapy was administered using 6-10 MV X-rays. All patients received three-dimensional conformal radiotherapy at a dose of 1.8-2.0 Gy per fraction, five times a week. The patients underwent radiotherapy for 4-6 weeks, receiving a total dose of 47-70 Gy. The primary gross tumor volume (GTV) and the volume of involved lymph nodes (GTV-N) were determined with the appliance of computerized imaging. The conformal clinical target volume (CTV) included the GTV with a $3 \mathrm{~cm}$ margin in the cephalad and caudad direction and a 0.5 $\mathrm{cm}$ margin in the lateral and anteroposterior directions. The CTV for lymph nodes included the GTV-N without an additional margin. The planning target volume (PTV) included the CTV with a $2 \mathrm{~cm}$ margin in the superior-inferior direction and a $0.5 \mathrm{~cm}$ margin in the lateral direction. ${ }^{12}$

Concurrent chemotherapy was administered using regimens that mainly included cisplatin plus 5 -fluorouracil or cisplatin plus docetaxel. In all, 78 patients were treated with two cycles of $60 \mathrm{mg} / \mathrm{m}^{2}$ docetaxel and $80 \mathrm{mg} / \mathrm{m}^{2}$ cisplatin delivered on days 1 and 22 of radiotherapy. ${ }^{13}$ Another 84 patients were treated with two cycles of $60 \mathrm{mg} / \mathrm{m}^{2}$ cisplatin administered on days 1 and 29 and $300 \mathrm{mg} / \mathrm{m}^{2} / 24 \mathrm{~h}$ 5-fluorouracil administered on days 1-3 and days 29-31. ${ }^{12}$ The remaining 50 patients received other chemotherapy regimens.

\section{Statistical analysis}

Continuous data are presented as mean \pm standard deviation (SD). The chi-square test, Student's $t$-test and Mann-Whitney $U$ test were used to compare the groups.

Overall survival (OS) and progression-free survival (PFS) were the study endpoints. OS was calculated from the day of diagnosis to the date of death or censorship at the date of last follow-up. PFS was calculated from the day of diagnosis to the detection of recurrent disease or to the date of death in patients without evidence of disease recurrence, or censorship at the date of last follow-up. Survival analysis was performed using the Kaplan-Meier method, and the differences were analyzed using the log-rank test. The three categories of mGPS $(0,1,2)$ were analyzed using pairwise comparisons, with the $p$-value set at 0.017 $(0.05 / 3)$ according to Hochberg's step-up method. ${ }^{14}$ Multivariate analyses using the Cox proportional hazards model were used to calculate hazard ratios and to test independent significance by backward elimination of non-significant explanatory variables. 
The criterion for statistical significance was set at $\alpha=$ $0.05, p$-values were determined using two-sided tests. All statistical analyses were performed using SPSS 16.0 software (SPSS Inc., Chicago, IL, USA).

\section{Results}

\section{Patient characteristics}

Of the 212 patients, 166 were male $(78.3 \%)$ and 46 were female $(21.7 \%)$. The median age of the patients was 60.0 years (range, 37-81 years). The demographic and clinical data of the patients are shown in Table 1. According to the 6th AJCC TNM classification, 26 patients were classified as stage I-II (12.3\%), 77 patients were classified as stage III (36.3\%) and 109 patients were classified as stage IV $(51.4 \%)$. The median duration of follow-up for patients who were alive at the time of the most recent follow-up was 35.0 months (range, 2-72 months).

\section{Correlation of mGPS with clinicopathological parameters}

Table 1 presents the correlations between the mGPS and various clinicopathological parameters. An increased mGPS was significantly associated with more advanced disease, including a larger tumor length (> $5 \mathrm{~cm}$ ), lymph node metastases (N1), distant metastases (M1), and stage III - IV disease (Table 1). No significant relationship was found between the mGPS and sex, age, histological type, tumor location or T stage.

The relationships between the mGPS and relevant continuous variables are shown in Table 2. Patients in the normal group (mGPS $=0$ ) had lower white blood cell counts, neutrophilic granulocyte counts and platelet counts compared to those in the abnormal group (mGPS $\geq 1$ ). In contrast, the mean total bilirubin and indirect bilirubin levels in the normal group $(\mathrm{mGPS}=0$ ) were higher than that of the abnormal group (mGPS $\geq 1$ ). There was no significant difference in the lymphocyte counts, hemoglobin, ALT, LDH and direct bilirubin levels between the normal group (mGPS $=0$ ) and abnormal group (mGPS $\geq 1$ ).

\section{Clinical predictors of survival}

The 3-year OS rate for the entire cohort was $24.6 \%$ and the 3 -year PFS rate was $21.3 \%$. The median OS time for all patients was 17.0 months and the median PFS time was 14.0 months.

Age, sex, tumor location, tumor length, clinical $\mathrm{T}$ stage, clinical $\mathrm{N}$ stage, $\mathrm{M}$ stage, radiation dose and stage were included in the univariate analysis. Several variables were significantly associated with OS: tumor location $(p=0.014)$, T stage $(p=0.014), \mathrm{N}$ stage $(p=$ $0.012)$, M stage $(p<0.001)$, mGPS $(p<0.001)$, albumin $(p<0.001)$ and CRP $(p<0.001)$. Tumor location $(p=$ $0.042)$, T stage $(p=0.014), \mathrm{N}$ stage $(p=0.020), \mathrm{M}$ stage $(p<0.001)$, mGPS $(p<0.001)$, albumin $(p=0.002)$, CRP $(p=0.001)$ and platelet count $(p=0.005)$ were significantly associated with PFS. The univariate survival analysis is presented in Table 3.

Table I. Demographic and pathological characteristics of the study population.

\begin{tabular}{|c|c|c|c|c|c|}
\hline Characteristic & Total (\%) & mGPS & & & $P$ \\
\hline & & 0 & 1 & 2 & \\
\hline Total & 212 & $90(42.5)$ & $78(36.8)$ & $44(20.8)$ & \\
\hline Age (years) & & & & & 0.223 \\
\hline$\leq 60$ & 114 & $49(43.0)$ & 37 (32.5) & $28(24.5)$ & \\
\hline$>60$ & 98 & $41(41.8)$ & 41 (41.8) & $16(16.4)$ & \\
\hline Sex & & & & & 0.201 \\
\hline Male & $166(78.3)$ & $65(39.2)$ & $64(38.5)$ & $37(22.3)$ & \\
\hline Female & $46(21.7)$ & $25(54.3)$ & $14(30.4)$ & $7(15.2)$ & \\
\hline Location & & & & & 0.892 \\
\hline Upper third & $85(40.1)$ & $39(45.9)$ & $31(36.5)$ & 15 (17.6) & \\
\hline Middle third & $104(49.1)$ & $42(40.4)$ & 38 (36.5) & $24(23.1)$ & \\
\hline Lower third & $23(10.8)$ & $9(39.1)$ & $9(39.1)$ & $5(21.8)$ & \\
\hline Tumor length & & & & & 0.000 \\
\hline$\leq 5 \mathrm{~cm}$ & $112(52.8)$ & $58(51.8)$ & $33(29.4)$ & $21(18.8)$ & \\
\hline$>5 \mathrm{~cm}$ & $100(47.2)$ & $32(32.0)$ & $45(45.0)$ & $23(23.0)$ & \\
\hline T stage & & & & & 0.079 \\
\hline T1-T2 & 37 (17.5) & $21(56.8)$ & $12(32.4)$ & $4(10.8)$ & \\
\hline $\mathrm{T} 3$ & $108(50.9)$ & $48(44.4)$ & 35 (32.4) & $25(23.2)$ & \\
\hline $\mathrm{T} 4$ & 67 (31.6) & $21(31.4)$ & $31(46.2)$ & $15(22.4)$ & \\
\hline N stage & & & & & 0.006 \\
\hline N0 & $18(8.5)$ & $14(77.8)$ & 2 (11.1) & 2 (11.1) & \\
\hline N1 & 194 (91.5) & $76(39.2)$ & $76(39.2)$ & 42 (21.6) & \\
\hline M stage & & & & & 0.000 \\
\hline M0 & 103 (48.6) & $56(54.4)$ & 37 (35.9) & $10(9.7)$ & \\
\hline M1 & 109 (51.4) & $34(31.2)$ & $41(37.6)$ & $34(31.2)$ & \\
\hline Stage & & & & & 0.001 \\
\hline I-II & $26(12.3)$ & $16(61.5)$ & $8(30.8)$ & $2(7.7)$ & \\
\hline III & 77 (36.3) & $40(51.9)$ & 29 (37.6) & $8(10.5)$ & \\
\hline IV & $109(51.4)$ & $34(31.2)$ & $41(37.6)$ & $34(31.2)$ & \\
\hline Radiation dose & & & & & 0.335 \\
\hline$<60 \mathrm{~Gy}$ & $70(33.0)$ & $25(35.7)$ & $30(42.8)$ & $15(21.5)$ & \\
\hline$\geq 60 \mathrm{~Gy}$ & $142(67.0)$ & $65(45.8)$ & $48(33.8)$ & $29(20.4)$ & \\
\hline
\end{tabular}

Table 2. Relationships between the mGPS and relevant continuous variables.

\begin{tabular}{|c|c|c|c|}
\hline & mGPS $=0$ & mGPS $\geq 1$ & $P$ \\
\hline White blood cells (WBC, count/L) & $7.0 \pm 1.8$ & $8.3 \pm 2.6$ & $<0.001$ \\
\hline $\begin{array}{l}\text { Neutrophilic granulocytes } \\
\text { (count/L) }\end{array}$ & $4.3 \pm 1.5$ & $5.4 \pm 2.1$ & $<0.001$ \\
\hline Lymphocytes (count/L) & $1.9 \pm 0.5$ & $1.9 \pm 0.8$ & 0.565 \\
\hline Hemoglobin $(\mathrm{g} / \mathrm{L})$ & $138.4 \pm 15.8$ & $135.1 \pm 15.8$ & 0.132 \\
\hline Platelets (count/L) & $228.0 \pm 61.1$ & $266.8 \pm 92.1$ & 0.001 \\
\hline $\begin{array}{l}\text { Aspartate aminotransferase (AST, } \\
\mathrm{U} / \mathrm{L} \text { ) }\end{array}$ & $22.3 \pm 10.9$ & $20.48 \pm 7.5$ & 0.135 \\
\hline $\begin{array}{l}\text { Alanine aminotransferase (ALT, } \\
\mathrm{U} / \mathrm{L} \text { ) }\end{array}$ & $19.4 \pm 11.6$ & $19.2 \pm 13.9$ & 0.935 \\
\hline Total bilirubin (mg/dL) & $12.9 \pm 5.8$ & $11.3 \pm 5.0$ & 0.029 \\
\hline Indirect bilirubin $(\mathrm{mg} / \mathrm{dL})$ & $9.0 \pm 4.0$ & $7.6 \pm 3.7$ & 0.012 \\
\hline Direct bilirubin $(\mathrm{mg} / \mathrm{dL})$ & $3.9 \pm 2.6$ & $3.6 \pm 1.5$ & 0.334 \\
\hline Lactate dehydrogenase (LDH, U/L) & $173.0 \pm 28.2$ & $172.6 \pm 42.0$ & 0.937 \\
\hline
\end{tabular}


Table 3. Univariate analysis of prognostic factors in patients with esophageal SCC.

\begin{tabular}{lllll}
\hline Variable & 3-year OS & $\boldsymbol{P}$ & 3-year PFS & $\boldsymbol{P}$ \\
\hline & $(\%)$ & & $(\%)$ & \\
Age $(\leq 60 />60$ years) & $26.0 / 20.6$ & 0.363 & $23.8 / 16.4$ & 0.340 \\
Sex (male/female) & $23.6 / 22.9$ & 0.959 & $21.5 / 19.4$ & 0.749 \\
Location (upper/middle, & $29.2 / 19.7$ & 0.014 & $26.1 / 16.6$ & 0.042 \\
lower) & & & & \\
Tumor length ( $\leq 5 />5 \mathrm{~cm})$ & $24.3 / 18.5$ & 0.239 & $24.2 / 17.2$ & 0.073 \\
T stage (T1-2/T3-4) & $33.0 / 20.9$ & 0.014 & $29.5 / 18.4$ & 0.014 \\
N stage (N0/N1) & $37.5 / 21.0$ & 0.012 & $32.4 / 18.6$ & 0.020 \\
M stage (M0/M1) & $36.2 / 10.9$ & $<0.001$ & $33.3 / 8.2$ & $<0.001$ \\
Radiation dose $(<60 / \geq 60$ & $25.3 / 28.1$ & 0.107 & $15.7 / 24.3$ & 0.150 \\
Gy) & & & & \\
mGPS $(0 / 1 / 2)$ & $34.6 / 21.7 / 4.5<0.001$ & $29.9 / 16.5 / 0.0$ & $<0.001$ \\
Albumin $(\geq 35 /<35 \mathrm{~g} / \mathrm{L})$ & $28.6 / 4.5$ & $<0.001$ & $24.6 / 4.5$ & 0.002 \\
CRP $(<10 / \geq 10 \mathrm{mg} / \mathrm{L})$ & $33.8 / 15.6$ & $<0.001$ & $28.0 / 13.5$ & 0.001 \\
Hemoglobin $(\geq 12 /<12$ & $32.1 / 23.4$ & 0.660 & $28.6 / 19.1$ & 0.578 \\
g/dL) & & & & \\
Plt $(\geq 200,000 /<200,000 / d L)$ & $29.4 / 22.5$ & 0.051 & $23.9 / 18.5$ & 0.019 \\
TB $(\leq 1.1 />1.1 \mathrm{mg} / \mathrm{dL})$ & $22.6 / 24.7$ & 0.138 & $17.7 / 20.7$ & 0.131 \\
LDH $(\geq 170 /<170 \mathrm{U} / \mathrm{L})$ & $30.0 / 18.2$ & 0.085 & $25.3 / 14.2$ & 0.193 \\
\hline
\end{tabular}

Plt : platelet; TB : total bilirubin; LDH: lactate dehydrogenase.

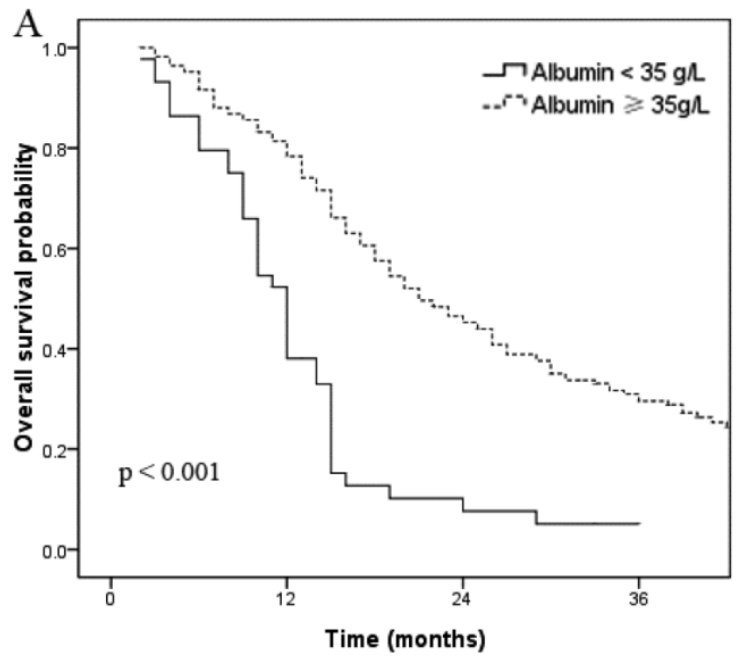

In this cohort, 54 patients $(20.7 \%)$ had hypoalbuminemia (albumin $<35 \mathrm{~g} / \mathrm{L}$ ). The 3-year OS rates for patients without and with hypoalbuminemia were $28.6 \%$ and $4.5 \%$, respectively $(p<0.001$, Fig. 1 a). The 3-year PFS rates for patients without and with hypoalbuminemia were $24.6 \%$ and $4.5 \%$, respectively $(p<$ 0.001 , Fig. 1b). A total of 122 patients had elevated CRP ( $\geq 10 \mathrm{mg} / \mathrm{L}$ ); univariate analysis indicated these patients had shorter OS and PFS times compared to the normal CRP group. The 3-year OS rate for patients with elevated CRP was $15.6 \%$ compared to $33.8 \%$ for patients with normal CRP $(p<0.001$, Fig. 2a). The 3-year PFS rates for patients with elevated CRP and normal CRP were $13.5 \%$ and $28.0 \%$, respectively $(p<$ 0.001, Fig. 2b).

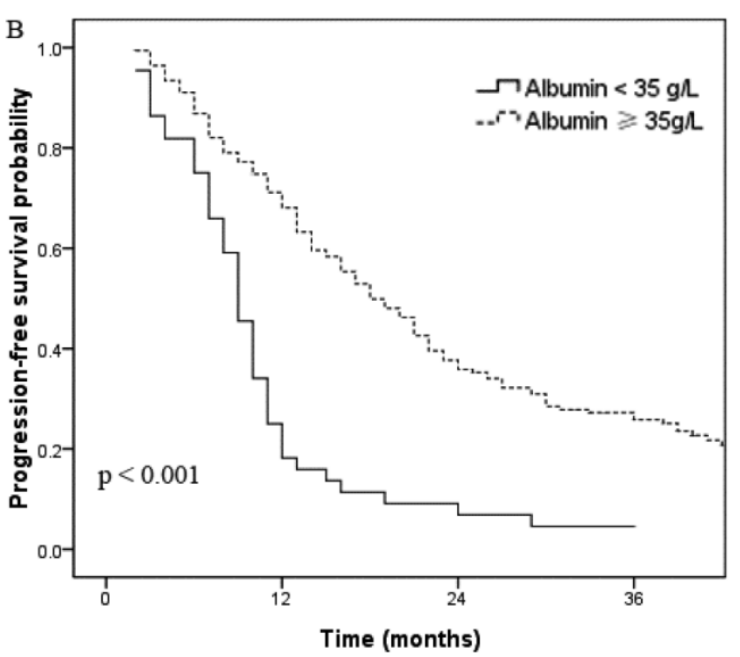

Figure I. Overall survival $(\mathrm{A})$ and progression-free survival $(\mathrm{B})$ curves for patients with normal and low $(<35 \mathrm{~g} / \mathrm{L})$ pretreatment serum albumin.
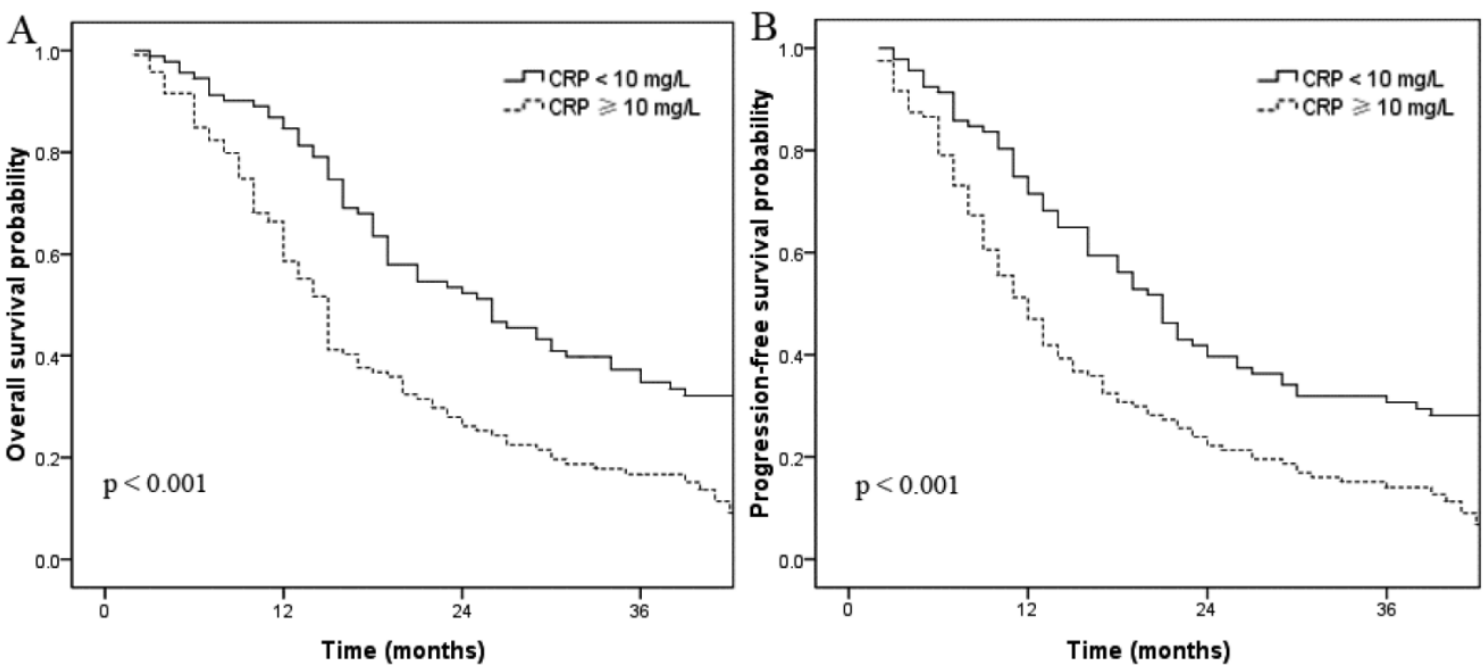

Figure 2. Overall survival $(A)$ and progression-free survival (B) curves for patients with serum $C R P \geq 10 \mathrm{mg} / \mathrm{L}$ and $<10 \mathrm{mg} / \mathrm{L}$. 
Elevated serum CRP and hypoalbuminemia were associated with shorter OS and PFS in univariate analysis. When these variables were scored as a combined index (mGPS), the total score provided good stratification of the patients in terms of survival $(p<$ 0.001 , Fig. 3). The 3-year OS rates for patients with a mGPS of 0,1 and 2 were $34.6 \%, 21.7 \%$ and $4.5 \%$, respectively. The 3-year PFS rates for patients with a mGPS of 0,1 and 2 were $29.9 \%, 16.5 \%$ and $0.0 \%$, respectively. An increase in the mGPS correlated significantly with gradual reductions in PFS and OS. Pairwise comparisons between the three subgroups (mGPS of 0,1 and 2) revealed that patients with a mGPS of 0 had significantly better 3-year OS $(p=$ $0.007)$ and PFS $(p=0.021)$ rates than patients with a mGPS of 1 . Patients with a mGPS of 0 also had significantly better 3-year OS $(p<0.001)$ and PFS $(p<$ $0.001)$ rates than patients with a mGPS of 2. Additionally, patients with a mGPS of 1 had significantly better 3-year OS $(p<0.001)$ and PFS $(p<0.001)$ rates than patients with a mGPS of 2 .

To identify independent prognostic factors, the factors that were found to be significant in univariate analysis were included in multivariate analysis. As there is duplication between albumin, CRP and the mGPS, only the mGPS was entered into the multivariate analysis. Multivariate analysis revealed that clinical $\mathrm{T}$ stage (HR 1.290, 95\% CI $1.023-1.627 ; p=$ 0.031 ), $M$ stage (HR 1.587, 95\% CI $1.138-2.215 ; p=$ 0.007) and mGPS (HR 1.694, 95\% CI $1.350-2.126$; $p<$ 0.001 ) were independent factors affecting OS in patients with esophageal SCC. Clinical T stage (HR $1.252,95 \%$ CI $1.001-1.565 ; p=0.049$ ), M stage (HR 1.827, 95\% CI 1.315 - 2.538; $p<0.001$ ), mGPS (HR 1.555, 95\% CI $1.243-1.947 ; p<0.001)$ and platelet count (HR 1.491, 95\% CI $1.014-2.193 ; p=0.042$ ) were independent factors affecting PFS in patients with esophageal SCC (Table 4).

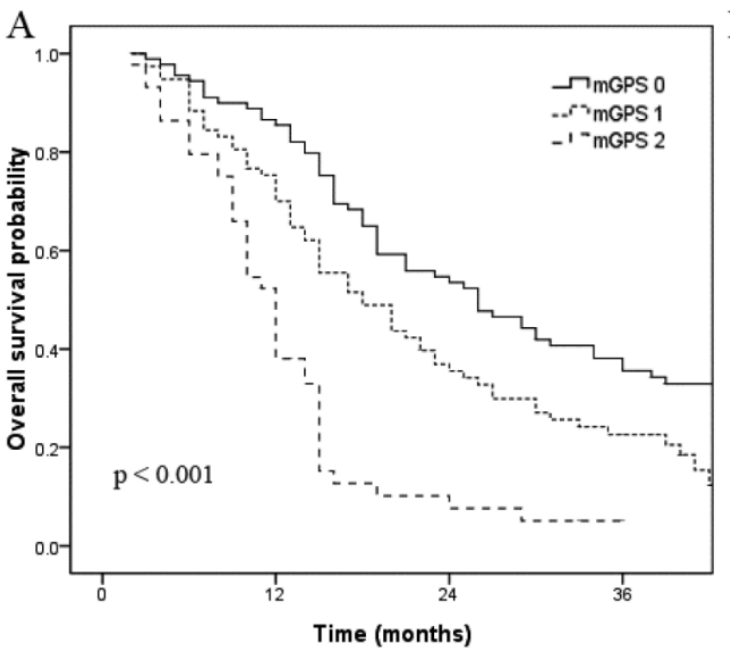

Table 4. Multivariate analysis of factors influencing OS and PFS in patients with esophageal SCC.

\begin{tabular}{lllll}
\hline Endpoint & Variable & $\boldsymbol{P a}^{\mathbf{a}}$ & HR & $\mathbf{9 5 \%}$ CI \\
\hline OS & Location & 0.063 & 1.254 & $0.988-1.591$ \\
& T stage & 0.031 & 1.290 & $1.023-1.627$ \\
& N stage & 0.247 & 1.465 & $0.768-2.798$ \\
& M stage & 0.007 & 1.587 & $1.138-2.215$ \\
PFS & mGPS & $<0.001$ & 1.694 & $1.350-2.126$ \\
& Location & 0.487 & 1.085 & $0.861-1.368$ \\
& T stage & 0.049 & 1.252 & $1.001-1.565$ \\
& N stage & 0.310 & 1.377 & $0.743-2.554$ \\
& M stage & $<0.001$ & 1.827 & $1.315-2.538$ \\
& mGPS & $<0.001$ & 1.555 & $1.243-1.947$ \\
& Platelets & 0.042 & 1.491 & $1.014-2.193$ \\
\hline
\end{tabular}

95\% CI, 95\% confidence interval; HR, hazard ratio; OS, overall survival; PFS, progression-free survival; SCC, squamous cell carcinoma.

a $P$ values were calculated using an adjusted Cox proportional hazards model.

\section{Discussion}

There is very little data available on the prognostic value of the mGPS in patients with inoperable SCC. This retrospective study of 212 patients revealed that the mGPS is an independent prognostic factor in patients with unresectable SCC undergoing chemoradiotherapy.

The mGPS is based on a combination of elevated CRP (> $10 \mathrm{mg} / \mathrm{L}$ ) and hypoalbuminemia (<35 g/L), and is indicative of both an underlying systemic inflammatory response and a nutritional decline. The GPS has been reported to be a useful predictor of survival in patients with cancer. However, a previous study indicated that a GPS of 1 was most commonly found to be the result of elevated CRP. Therefore, the GPS was modified so that patients with hypoalbuminemia in the absence of elevated CRP were assigned a score of $0 . .^{15}$ The mGPS has been shown to have prognostic value in patients with lung, gastrointestinal, and colorectal cancer. ${ }^{16-19}$

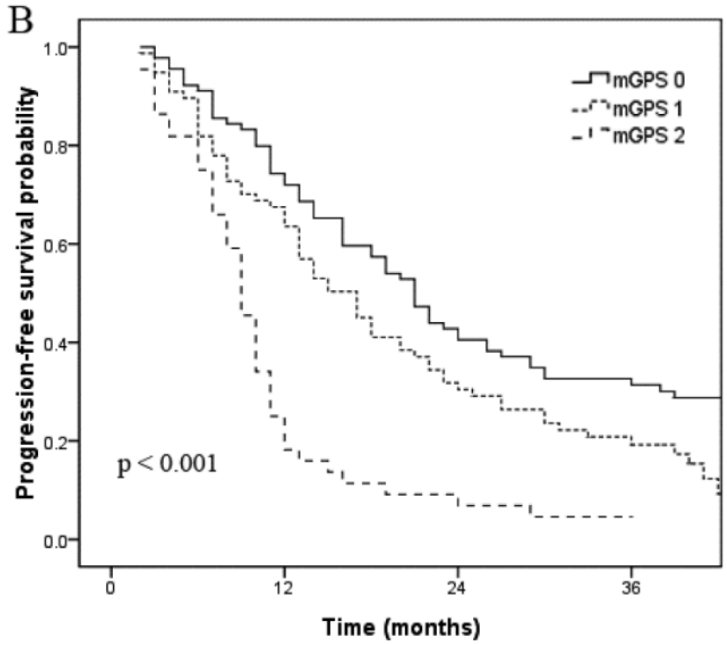

Figure 3. Overall survival (A) and progression-free survival (B) curves for patients with a mGPS of $0, \mathrm{I}$, and 2 . 
Previous reports have shown that systemic inflammation, as indicated by elevated CRP, strongly influenced the prognosis of patients with gastrointestinal carcinomas, including gastro-esophageal cancer, ${ }^{19}$ pancreatic cancer ${ }^{20}$ and colorectal cancer ${ }^{18}$. Marsik et al. examined the relationship between CRP and all-cause mortality in approximately 270,000 patients with cancer. As CRP increased from normal $(<5$ $\mathrm{mg} / \mathrm{L}$ ) to highly elevated ( $>80 \mathrm{mg} / \mathrm{L}$ ), there was a 1.4 -fold (5-10 mg/L category) to 3.3-fold (> $80 \mathrm{mg} / \mathrm{L}$ category) increase in the risk of all-cause mortality. ${ }^{21}$ In this study, elevated serum CRP correlated with poorer OS and PFS in patients with esophageal SCC (3 year OS: $p<0.001 ; 3$ year PFS: $p<0.001)$. The mechanisms by which systemic inflammation negatively influences survival are affected by numerous confounding factors. Firstly, elevated CRP levels can accelerate angiogenesis based on increased level of circulating levels of vascular growth factors and circulating interleukin in the gastroesophageal cancer patients. ${ }^{22}$, 23 Secondly, a systemic inflammatory response can impair the T-lymphocytic response to cancer, while the intratumoral CD8 (+) T cell infiltrations is a favorable prognostic factor by promoting proliferative activity and IFN- $\gamma$ secretion. ${ }^{24}$ Additionally, elevated acute-phase response proteins, especially CRP, are associated to cancer-related cachexia. ${ }^{23}$ Besides, the concomitant nutritional decline reduces patient tolerance to treatment toxicities and patient compliance with treatment declines. ${ }^{25}$

A marked elevation of serum CRP (> $100 \mathrm{mg} / \mathrm{L}$ ) can also be indications of acute bacterial infections, however, patients with cancer have persistently moderately elevated serum CRP levels $1<100$ $\mathrm{mg} / \mathrm{L}){ }^{26}$ It has been suggested that a non-specific inflammatory response secondary to tumor hypoxia and necrosis or local tissue damage occurs in patients with cancer. ${ }^{27}$

In parallel to elevated serum CRP, reduced serum albumin has been observed and verified in patients with various types of cancer. ${ }^{28}$ The development of hypoalbuminemia is often secondary to a systemic inflammatory response. ${ }^{29}$ McMillan et al. also reported that hypoalbuminemia alone reflected a systemic inflammatory response. ${ }^{28}$ In this study, hypoalbuminemia correlated with poorer OS and PFS in patients with esophageal SCC (3 year OS: $p<0.001 ; 3$ year PFS: $p<0.001)$. This result may be attributable to a state of impaired immunity and poor nutrition in patients with inoperable SCC patients.

The mGPS was an independent prognostic factor for OS and PFS in patients with inoperable thoracic esophageal SCC in multivariate analysis (3 year OS: $p<0.001$; 3 year PFS: $p<0.001$ ). Heo et al. compared the four post hoc multiple pairwise testing methods, and concluded that the Hochberg method has a low false discovery rate and the highest correct decision rate of the four methods. ${ }^{14}$ Post hoc pairwise comparisons between the three groups showed the OS and PFS rates of patients with a GPS of 1 were significantly different to patients with a GPS of 0 or 2, and there was a good stratification of the OS and PFS rates of the patients in the three groups. Therefore, the mGPS offers a simple, objective and well standardized pre-treatment assessment which can aid in the selection of the optimum treatment strategy in patients with inoperable thoracic esophageal SCC.

Multivariate analysis revealed that the major significant predictive factors for OS in patients with inoperable thoracic esophageal SCC were T stage, $\mathrm{M}$ stage and the mGPS; similar factors were also significant for PFS.

Although the mGPS had significant prognostic value for long-term OS and PFS in patients with inoperable thoracic esophageal SCC, this study is biased by its retrospective nature. However, a large, homogeneous population that only underwent chemoradiotherapy was analyzed in this study.

\section{Conclusion}

The prognosis for patients with inoperable esophageal SCC undergoing chemoradiotherapy remains poor. The pretreatment mGPS is an easily measurable biomarker with prognostic significance in these patients. The mGPS can be used in combination with conventional TNM staging to predict survival in patients with esophageal SCC undergoing chemoradiotherapy.

\section{Acknowledgement}

This work was supported by the grant from the Sci-Tech Project Foundation of Guangdong Province (No. 2012B031800287).

\section{Conflict of Interest}

The authors have declared no conflicts of interest.

\section{References}

1. Siegel R, Naishadham D, Jemal A. Cancer Statistics, 2013. CA Cancer J Clin. 2003; 63: 11-30.

2. Schneider BJ, Urba SG. Preoperative chemoradiation for the treatment of locoregional esophageal cancer: the standard of care? Semin Radiat Oncol. 2007;17:45-52.

3. Fujiwara $\mathrm{H}$, Suchi $\mathrm{K}$, Okamura $\mathrm{S}$, et al. Elevated serum CRP levels after induction chemoradiotherapy reflect poor treatment response in association with IL-6 in serum and local tumor site in patients with advanced esophageal cancer. J Surg Oncol. 2011; 103: 62-68.

4. Vashist YK, Loos J, Dedow J, et al. Glasgow Prognostic Score is a predictor of perioperative and long-term outcome in patients with only surgically treated esophageal cancer. Ann Surg Oncol. 2011;18(4):1130-8.

5. Wang CY, Hsieh MJ, Chiu YC, et al. Higher serum C-reactive protein concentration and hypoalbuminemia are poor prognostic indicators in patients with esophageal cancer undergoing radiotherapy. Radiother Oncol. 2009; 92(2):270-5. 
6. O'Gorman P, McMillan DC, McArdle CS. Longitudinal study of weight, appetite, performance status, and inflammation in advanced gastrointestinal cancer. Nutr Cancer. 1999; 35(2): 127-9.

7. O'Gorman P, McMillan DC, McArdle CS. Prognostic factors in advanced gastrointestinal cancer patients with weight loss. Nutr Cancer. 2000; 37(1):36-40.

8. Ishizuka $\mathrm{M}$, Nagata $\mathrm{H}$, Takagi $\mathrm{K}$, et al. Inflammation-based prognostic score is a novel predictor of postoperative outcome in patients with colorectal cancer. Ann Surg. 2007; 246:1047-51.

9. Nozoe T, Iguchi T, Egashira A, et al. Significance of modified Glasgow prognostic score as a useful indicator for prognosis of patients with gastric carcinoma. Am J Surg. 2011; 201:186-91.

10. Glen P, Jamieson NB, McMillan DC, et al. Evaluation of an inflammation-based prognostic score in patients with inoperable pancreatic cancer. Pancreatology. 2006; 6:450-3.

11. Proctor MJ, Morrison DS, Talwar D, et al. An inflammation-based prognostic score (mgps) predicts cancer survival independent of tumour site: a glasgow inflammation outcome study. Br J Cancer. 2011; 104:726-34.

12. Liu H, Lu L, Zhu Q, et al. Cervical nodal metastases of unresectable thoracic esophageal squamous cell carcinoma Characteristics of long-term survivors after concurrent chemoradiotherapy. Radiother Oncol. 2011; 99 (2):181-6.

13. Li QQ, Liu MZ, Hu YH, et al. Definitive concomitant chemoradiotherapy with docetaxel and cisplatin in squamous esophageal carcinoma. Dis Esophagus. 2010; 23:253-9.

14. Heo M, Leon AC. Comparison of post hoc multiple pairwise testing procedures as applied to small k-group logrank tests. Int J Statist Med Res. 2013; 2: 104-116.

15. McMillan DC, Crozier JE, Canna K et al. Evaluation of an inflammation-based prognostic score (GPS) in patients undergoing resection for colon and rectal cancer. Int J Color Dis. 2007; 22:881-886

16. Crumley AB, McMillan DC, McKernan M, et al. Evaluation of an inflammation-based prognostic score in patients with inoperable gastro-oesophageal cancer. Br J Cancer. 2006; 94(5):637-41.

17. Jeong JH1, Lim SM, Yun JY, et al. Comparison of two inflammation-based prognostic scores in patients with unresectable advanced gastric cancer. Oncology. 2012; 83(5):292-9.

18. McMillan DC, Crozier JE, Canna K, et al. Evaluation of an inflammation-based prognostic score (GPS) in patients undergoing resection for colon and rectal cancer. Int J Colorectal Dis. 2007; 22(8):881-6.

19. Crumley AB, McMillan DC, McKernan M, et al. An elevated C-reactive protein concentration, prior to surgery, predicts poor cancer-specific survival in patients undergoing resection for gastro-oesophageal cancer. Br J Cancer. 2006; 94:1568-71.

20. La Torre M, Nigri G, Cavallini M, et al. The glasgow prognostic score as a predictor of survival in patients with potentially resectable pancreatic adenocarcinoma. Ann Surg Oncol. 2012; 19:2917-23.

21. Marsik C, Kazemi-Shirazi L, Schickbauer T, et al. C-reactive protein and allcause mortality in a large hospital-based cohort. Clin Chem 2008; 54:343349.

22. McMillan DC. Systemic inflammation, nutritional status and survival in patients with cancer. Curr Opin Clin Nutr Metab Care. 2009;12(3):223-6.

23. Krzystek-Korpacka M, Matusiewicz M, Diakowska D, et al. Acute-phase response proteins are related to cachexia and accelerated angiogenesis in gastroesophageal cancers. Clin Chem Lab Med. 2008; 46(3):359-64.

24. Schumacher K, Haensch W, Roefzaad C, et al. Prognostic significance of activated CD8(+) T cell infiltrations within esophageal carcinomas. Cancer Res. 2001; 61:3932-6.

25. Scott HR, McMillan DC, Forrest LM, et al. The systemic inflammatory response, weight loss, performance status and survival in patients with inoperable non-small cell lung cancer. Br J Cancer. 2002; 87:264-7.

26. Kushner I, Rzewnicki D, Samols D. What does minor elevation of C-reactive protein signify? Am J Med. 2006; 119: e117-128.

27. Thompson CB. Apoptosis in the pathogenesis and treatment of disease. Science. 1995; 267: 1456-1462.

28. McMillan DC. An inflammation-based prognostic score and its role in the nutrition-based management of patients with cancer. Proc Nutr Soc. 2008;67(3):257-62.

29. McMillan DC, Elahi MM, Sattar N, et al. Measurement of the systemic inflammatory response predicts cancer-specific and non-cancer survival in patients with cancer. Nutr Cancer. 2001; 41:64-9. 